\title{
A new twist in the regulation of Legionella replication through ASC and caspase-1
}

\author{
Luigi Franchi and Gabriel Núñez* \\ Department of Pathology and Comprehensive Cancer Center, University of Michigan Medical School, Ann Arbor, MI, USA \\ ${ }^{*}$ Correspondence: bclx@umich.edu
}

The recognition of bacteria is mediated by host pattern recognition receptors (PRRs) that detect conserved microbial structures and activate immune responses against the pathogen. These PRRs include membrane-bound toll-like receptors (TLRs) and cytosolic nod-like receptors (NLRs). In response to bacterial recognition, TLRs and several NLRs induce transcriptional induction of inflammatory and antimicrobial genes as well as type I IFNs. In addition, a set of NLRs including NLRP1, NLRP3, and NLRC4 is responsible for triggering the activation of the inflammasome, a molecular platform that drives the activation of caspase-1, a protease responsible for the maturation and secretion of IL- $1 \beta$ and IL-18 (Franchi et al., 2009). A feature of the inflammasomes is the use of the adaptor ASC (apoptosis-related speck-like protein) to link NLRs to pro-caspase-1, a critical step in the activation of caspase-1.

Legionella pneumophila (Legionella) is a Gram-negative facultative intracellular bacterium which causes an acute form of pneumonia called Legionnaires' disease. After infection of macrophages, bacteriacontaining phagosomes are converted into specialized Legionella-containing vacuoles (LCV) where the bacterium replicates. Interestingly, while human macrophages are permissive, most inbred mouse strains, with the exception of the $\mathrm{A} / \mathrm{J}$ mouse strain, are non-permissive for intracellular proliferation of L. pneumophila. Previous studies revealed several PPRs that are involved in the detection of Legionella. Among them, TLR5, NLRC4, and Naip5, are activated though the recognition of flagellin, a major component of the flagellum. While TLR5 recognizes flagellin during the engulfment of Legionella, NLRC4 and Naip5 sense flagellin released into the cytosol. In mouse macrophages cytosolic flagellin promote the assembly of the NLRC4 inflammasome inducing the activation of caspase- 1 and secretion of IL- $1 \beta$ and IL-18. Notably, NLRC4-dependent caspase-1 activation was found to restrict the intracellular growth of Legionella, at least in part, by promoting the fusion of the LCV with lysosomes (Amer et al., 2006). In mouse macrophages, the regulation of intracellular Legionella growth by the NLRC4 inflammasome is independent of IL-1 $\beta$ and IL-18, but relies on caspase-7, another protein substrate of caspase-1 (Akhter et al., 2009). In addition, Naip5 contributes to the recognition of the C-terminal portion of flagellin; however, Naip5 can restrict Legionella growth independently of the NLRC4 inflammasome because full length flagellin induces caspase- 1 activation in the absence of Naip5 (Lightfield et al., 2011). In mouse macrophages, NLRC4 and Naip5 also restrict pathogen growth by the induction of macrophage cell death under conditions of high multiplicity of infection (Case et al., 2009).

The role of ASC in controlling Legionella replication has turned out to be complex. The adaptor ASC regulates not only caspase- 1 activation, but also cell death and the transcription factor NF- $\mathrm{KB}$ by mechanisms that remain poorly understood. Recent work from Amer et al. (2006) have provided insight into the role of ASC in the control of Legionella replication in human monocytes and mouse macrophages (Abdelaziz et al., 2011a,b). A main difference between human monocytes and mouse macrophages is that human monocytes, but not mouse macrophages from many strains including C57BL6, allow replication of Legionella. In an attempt to understand this differential response, Amer et al. (2006) evaluated the inflammasome and found that unlike in non-permissive mouse macrophages, Legionella did not induce caspase-1 activation in human monocytes. The lack of caspase-1 activation could be explained by the ability of Legionella to inhibit the expression of NLRC4 and, to a lesser extent, ASC. To confirm that the downregulation of ASC had a role in preventing caspase- 1 activation, they transfected primary monocytes with a ASC-producing plasmid and found that the expression of ASC restored caspase- 1 activation and restriction of Legionella replication. As Legionella does not induce caspase-1 activation in human monocytes, Amer et al. (2006) also assessed whether ASC was involved in restricting Legionella replication independent of its role on caspase- 1 and found that depletion of ASC by siRNA promoted activation of NF- $\mathrm{\kappa B}$ and enhanced bacterial growth in human monocytes. In summary, this work reveals a mechanism by which Legionella evades recognition by the inflammasome and new function of ASC in restricting pathogen replication in human monocytes.

Amer et al. (2006) also investigated the role of ASC in mouse macrophages where activation of the caspase-1/caspases-7 cascade restricts Legionella replication. It was found that while NLRC4-deficient macrophages failed to restrict bacterial growth, ASC-deficient macrophages were capable of doing so as well as wild-type macrophages. The authors provided evidence that ASCdeficient macrophages can still induce caspase- 1 activation, albeit to a greatly reduced extent. Their results support the existence of a minor NLRC4-dependent, ASC-independent pathway of caspase- 1 activation that is sufficient to promote the fusion of the LCV to the lysosomes leading to inhibition of bacterial replication. In this model, ASC amplifies, but it is not necessary, for caspase-1 activation in the context of Legionella infection. Alternatively, it is possible that two different protein complexes with different function might be formed, one containing NLRC4, ASC, and caspase- 1 that is responsible for the secretion of IL-1 $\beta / \mathrm{IL}-18$ and the other formed by NLRC4 and caspase-1 that is responsible for restricting bacterial replication and/or inducing cell death (Broz et al., 2010; Figure 1A). As Legionella mutants lacking flagellin do not activate the NLRC4 inflammasome and replicate in wild-type mouse macrophages, the authors investigated whether ASC could restrict bacterial replication independent on its role in caspase-1 activation (Figure 1B). They found that 


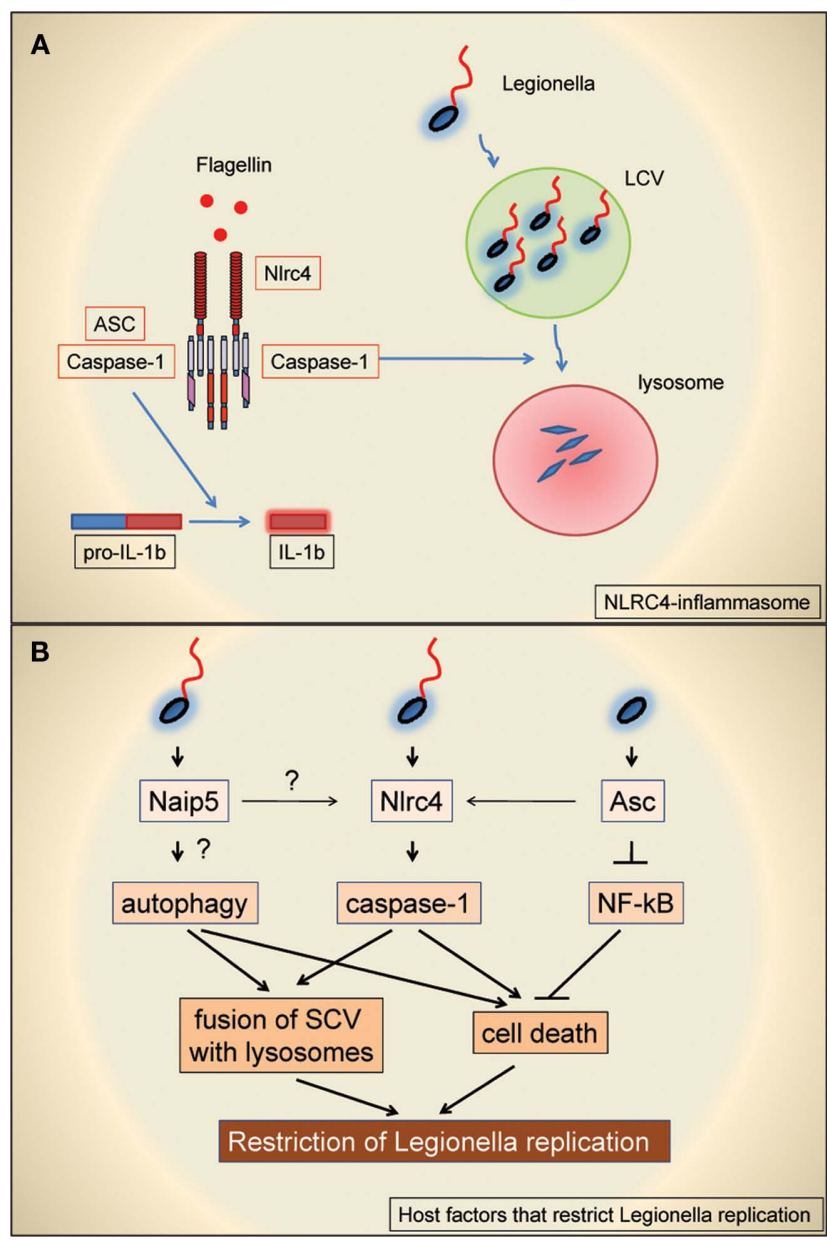

FIGURE 1 | (A) Schematic representation of the proposed function of NLRC4, ASC, and caspase-1 in the regulation of $L$. pneumophila replication in macrophages. The NLRC4-ASC-caspase-1 inflammasome promotes the maturation of pro-IL-1 $\beta$ whereas NLRC4-caspase-1 promotes the fusion of Legionellacontaining vacuoles (LCV) with lysosomes, leading to bacterial degradation. Note that in this model ASC is required for the maturation of $\mathrm{IL}-1 \beta$ but is not necessary to promote LCV fusion with lysosomes. (B) Schematic representation of host proteins involved in restricting Legionella replication in nonpermissive mouse macrophages and their proposed mode of action. See text for more details.

Legionella mutant lacking flagellin replicated more effectively in ASC-deficient cells, and this correlated with reduced cell death. Lastly, the authors showed that ASC was also able to restrict bacterial replication in caspase-1deficient cells and this was associated with reduced activation of NF- $\kappa \mathrm{B}$ and macrophage survival (Figure 1B). These results suggest that ASC controls Legionella replication through the regulation of cell survival which is only revealed in caspase-1-deficient macrophages. A potential problem with the interpretation of these experiments is that in this setting the higher number of intracellular bacteria may secondarily result in enhanced $\mathrm{NF}-\kappa \mathrm{B}$ activation. More research is needed to better understand the link between ASC and NF- $\kappa \mathrm{B}$ activation in controlling the permissiveness to Legionella replication.

In summary, the recent work by Amer et al. (2006) provide insight into the role of ASC in the activation of the NLRC4 inflammasome and reveal that ASC can restrict Legionella replication via different mechanisms including caspase- 1 activation, the modulation of $\mathrm{NF}-\kappa \mathrm{B}$ and the induction of cell death.

\section{ACKOWLEDGMENTS}

The authors research is funded by grants R01 AI0647748, R01 Al063331, and R01 DK61707 from the National Institutes of Health to Gabriel Núñez. Luigi Franchi is supported by a Career Development Award from the Crohn's and Colitis Foundation of America.

\section{REFERENCES}

Abdelaziz, D. H., Gavrilin, M. A., Akhter, A., Caution, K., Kotrange, S., Khweek, A. A., Abdulrahman, B. A., Grandhi, J., Hassan, Z. A., Marsh, C., Wewers, M. D., and Amer,A. O. (2011a).Apoptosis-associated specklike protein (ASC) controls Legionella pneumophila infection in human monocytes. J. Biol. Chem. 286, 3203-3208.

Abdelaziz, D. H., Gavrilin, M. A., Akhter, A., Caution, K., Kotrange,S., Khweek,A.A.,Abdulrahman, B.A.,Hassan, Z.A., El-Sharkawi, F.Z., Bedi, S. S., Ladner, K., Mostafa, M., Doseff, A., Gonzalez-Mejia, E. M., Guttridge, D., Kanneganti, T. D., Marsh, C. B., Wewers, M. D., and Amer, A. O. (2011b). Asc-dependent and independent mechanisms contribute to restriction of Legionella pneumophila infection in murine macrophages. Front. Microbiol. 2:18. doi: 10.3389/fmicb.2011.00018

Akhter, A., Gavrilin, M. A., Frantz, L., Washington, S., Ditty, C., Limoli, D., Day, C., Sarkar, A., Newland, C., Butchar, J., Marsh, C. B., Wewers, M. D., Tridandapani, S., Kanneganti, T.D., and Amer,A. O. (2009). Caspase-7 activation by the Nlrc4/Ipaf inflammasome restricts Legionella pneumophila infection. PLoS Pathog. 5, e1000361. doi: 10.1371/journal.ppat.1000361

Amer, A., Franchi, L., Kanneganti, T. D., Body-Malapel, M., Ozoren, N., Brady, G., Meshinchi, S., Jagirdar R., Gewirtz, A., Akira, S., and Nunez, G. (2006). Regulation of Legionella phagosome maturation and infection through flagellin and host Ipaf. J. Biol. Chem. 281, 35217-35223.

Broz, P., von Moltke, J., Jones, J.W., Vance, R. E., and Monack, D. M. (2010). Differential requirement for Caspase-1 autoproteolysis in pathogen-induced cell death and cytokine processing. Cell Host Microbe 8, 471-483.

Case, C. L., Shin, S., and Roy, C. R. (2009). Asc and Ipaf Inflammasomes direct distinct pathways for caspase-1 activation in response to Legionella pneumophila. Infect. Immun. 77, 1981-1991.

Franchi, L., Eigenbrod, T., Munoz-Planillo, R., and Nunez, G. (2009). The inflammasome: a caspase-1-activation platform that regulates immune responses and disease pathogenesis. Nat. Immunol. 10, 241-247.

Lightfield, K. L., Persson, J., Trinidad, N. J., Brubaker, S. W., Kofoed, E. M., Sauer, J. D., Dunipace, E. A., Warren, S. E., Miao, E. A., and Vance, R. E. (2011). Differential requirements for NAIP5 in activation of the NLRC4 (IPAF) inflammasome. Infect. Immun. doi:10.1128/ IAI.01187-10. [Epub ahead of print].

Received: 08 March 2011; accepted: 11 March 2011; published online: 21 March 2011.

Citation: Franchi L and Núñez G (2011) A new twist in the regulation of Legionella replication through ASC and caspase-1. Front. Microbio. 2:57. doi: 10.3389/ fmicb.2011.00057

This article was submitted to Frontiers in Cellular and Infection Microbiology, a specialty of Frontiers in Microbiology.

Copyright (C) 2011 Franchi and Núnez. This is an open-access article subject to an exclusive license agreement between the authors and Frontiers Media SA, which permits unrestricted use, distribution, and reproduction in any medium, provided the original authors and source are credited. 